\title{
Prevalence and type distribution of human papillomavirus infection among women with different degrees of cervical cytological abnormalities in Sicily (Italy)
}

\author{
Concetta Franchina, ${ }^{1,2}$ Carmela M. Costanzo, ${ }^{1}$ Raffaela Russo, ${ }^{1,2}$ Concetta I. Palermo, ${ }^{2}$ \\ Guido Scalia ${ }^{1,2}$
}
${ }^{1}$ Department of Biomedical and Biotechnological Sciences, University of Catania; ${ }^{2}$ Clinical Virology Unit, Policlinic Vittorio Emanuele, Gaspare Rodolico Hospital, Catania, Italy

\section{Summary}

Human papillomaviruses (HPVs) are etiological agents of cervical cancer. In the absence of Pap smear alterations, high-risk HPV DNA can be detected in cervical samples. The prevalence of papillomavirus

Correspondence: Concetta Franchina, Clinical Virology Unit, A.O.U. Policlinico-Vittorio Emanuele, P.O. Gaspare Rodolico, Via S. Sofia, 78, 95123 Catania, Italy.

Tel./Fax: +39.095.3782807

E-mail: concyfranchina@yahoo.it

Key words: Papillomavirus infection; HR-HPV; LR-HPV; cervical cytology; multiple infections.

Acknowledgements: the authors wish to thank the Scientific Bureau of the University of Catania for language support. The study protocol was approved by the Ethics Committee of the University Hospital "Policlinico-Vittorio Emanuele" of Catania and it is conformed to the ethical guidelines of the 1975 Helsinki Declaration. There was no need for informed written consent as this was a retrospective review of data where all subjects were completely anonymous.

Contributions: CF designed the experiments, participated in performing the molecular tests, performed the interpretation of data, and drafted the manuscript; CMC conceived the study, performed the molecular tests and the interpretation of data, and drafted the manuscript; RR, CIP participated in interpretation of data and helped to draft the manuscript; GS participated in the design of the study, supervised and coordinated it, and participated in drafting the manuscript. All authors read and approved the final manuscript.

Conflict of interest: the authors declare no potential conflict of interest.

Received for publication: 23 Janaury 2016.

Revision received: 30 March 2016.

Accepted for publication: 31 March 2016.

(C) Copyright C. Franchina et al., 2016

Licensee PAGEPress, Italy

Microbiologia Medica 2016; 31:5018

doi:10.4081/mm.2016.5018

This article is distributed under the terms of the Creative Commons Attribution Noncommercial License (by-nc 4.0) which permits any noncommercial use, distribution, and reproduction in any medium, provided the original author(s) and source are credited. infection and their genotype distribution varies greatly across populations. The aims of this study were: i) to assess the prevalences of HPV genotypes in people living in Eastern Sicily (Italy) and the frequency of HPV multiple infections; ii) to evaluate the association between HPV genotypes and cervical lesions in order to improve the epidemiological knowledge useful for monitoring or treating infected women. Nested PCR and reverse dot/blot hybridization were used for the detection and typing of HPV DNA in 315 women who had had an abnormal PAP-smear. HPV DNA test was positive in $70.5 \%$ cases; the prevalence was $50 \%$ in atypical squamous cells of undetermined significance (ASCUS), $80.8 \%$ in low grade-, and $76.2 \%$ in high grade-squamous intraepithelial lesion (H-SIL). The genotype distribution showed a predominance of HPV-16 (56.7\%) followed by HPV-18 (12.2\%), HPV-31 (9.5\%) and HPV-6 (9.5\%). Multiple infections were detected in 35.1\% of the infected patients. High frequency of positive results for HPV was confirmed and, even in case of ASCUS, patients should be taken into account for genotyping. Our data indicate that multiple infections are consistent in women with low-grade lesions while they are less frequent in women with H-SIL. This could reinforce the theory of the multi-stage cancer model, by which one HPV type becomes predominant along with the progression of cervical lesion severity.

\section{Introduction}

Genital human papillomavirus (HPV) infection is the most common sexually transmitted viral infection worldwide (3). It is associated with various clinical conditions, ranging from asymptomatic infections to benign or malignant diseases of the genital mucosa (7).

More than 100 human papillomavirus (HPV) genotypes are known, and more than 40 of these can infect the anogenital area either in men or in women. On the basis of the phylogenetic and epidemiological characteristics, they are grouped as low-risk HPV (LR-HPV), commonly associated with low-grade cervical lesions, and high-risk HPV (HRHPV), associated with high-grade lesions and/or cancer $(5,28)$. Genotype distribution may vary globally and within geographical areas. In a Planned Parenthood population in the United States with a mean age of 25 years, the prevalence of high-risk HPV infection was $27.4 \%$ (17). Similar prevalence estimates were found among female university students in the U.S. and Canada (16). In Europe, the most prevalent genotype is HPV 16, followed by HPV 31 and HPV 66 in Eastern and Southern Europe, respectively (12). A recent study showed that in Scotland the prevalence of PCR-detected HPV DNA in women (mean age 36.6 years) attending routine cervical screening was approximately $20.5 \%$ for all HPVs and $15.7 \%$ for HR-HPVs (10). In a study run in 
Barcelona, HPV DNA was detected in $68 \%$ of a cohort of women followed due to cervical dysplasia (24).

At present, little is known about the prevalence and distribution of HPV genotypes in Italy. Only a few studies, mainly based on Northern Italy populations, have investigated the prevalence of cervical HPV infection in healthy women $(6,11,21)$ and in women with cytological abnormalities $(15,19,27)$.

The aims of this study were: i) to assess the prevalences of HPV genotypes and the frequency of multiple infections in people living in Eastern Sicily; ii) to evaluate the association between HPV genotypes and cervical lesions in order to improve the epidemiological knowledge useful for monitoring or treating infected women.

\section{Materials and Methods}

\section{Study population}

This is a prospective study that included a cohort of 315 women, who were referred to the Clinical Virology Unit of the Central Laboratory, University Hospital Policlinico-Vittorio Emanuele, G. Rodolico of Catania, Italy, from February 2009 to February 2010.

The mean age of the patients was 34.5 years (range, 18-65 years).

All women included in this study had had a PAP smear classified as abnormal according to the Bethesda System (TBS, 2001) that reports cervical cytological diagnoses: atypical squamous cells of undetermined significance (ASCUS), low grade squamous intraepithelial lesion (LSIL), and high grade squamous intraepithelial lesion (H-SIL). Thus, women were subdivided into three groups: 96 (30.5\%) were classified as ASCUS; 156 (49.5\%) as L-SIL; and 63 (20.0\%) as H-SIL.

\section{Sample collection}

Endocervical cytobrush or cervical swab was collected and suspended in $20 \mathrm{~mL}$ of ThinPrep ${ }^{\circledR}$ Pap Test PreservCyt ${ }^{\circledR}$ Solution (Hologic Inc, MA, USA). The solution was placed into two tubes and pelleted by centrifugation at $1000 \mathrm{rpm}$ for $20 \mathrm{~min}$. The supernatant was removed and discarded and the pellet was resuspended in $2 \mathrm{ml}$ aliquots in 2 tubes and stored at $-20^{\circ} \mathrm{C}$ until use.

\section{Human papillomavirus DNA extraction, amplification, and genotyping}

The automated DNA extraction was performed with $1 \mathrm{ml}$ of sample on the NucliSens easyMAG system (bioMérieux SA, Marcy l'Etoile, France) following the manufacturer's HPV 1.1 protocol, with a $55 \mu \mathrm{L}$ final elution volume.

Amplification of HPV DNA was accomplished by nested PCR using HPV-HS Bio kit (AB Analitica s.r.l, Padova, Italy) according to the manufacturer's recommendations.
For the first-amplification step, performed with $10 \mu \mathrm{L}$ of eluate, a combination of degenerate primers was used to amplify a 449-458 bp sequence within the L1 ORF of the HPV genome. The second amplification was performed from $1 \mu \mathrm{L}$ of the first amplification product, using biotinylated primers to amplify a $139-145$ bp sequence. To verify the efficiency of the DNA extraction, $10 \mu \mathrm{L}$ of eluate were used to amplify a 202 bp fragment of the Thiosulfate SulfurTransferase (TST) gene using specific primers. Negative (water) and positive controls (plasmid clones containing HPV 54) were included in each PCR run, to check for accuracy or possible contamination. The product of the second amplification and of the TST were detected and identified by electrophoretic separation into a $2 \%$ agarose gel with $2,5 \mathrm{mg} / \mathrm{ml}$ ethidium bromide in TAE buffer. A specimen was considered positive if a $202 \mathrm{bp}$ band (TST) and of 139-145 bp band (HPV target) were detected by an UV trransilluminator. The positive specimens were used for the hybridization step; those negative for TST were considered inadequate and extracted again from the second tube.

Finally, HPV typing was performed with a reverse line blot hybridization assay with specific probes for the most frequent HPV-types (Ampliquality HPV-type assay, AB Analitica s.r.l., Padova, Italy). HPV-type allows the identification of 11 LR-HPV (HPV-6, -11, -40, -42, -43, -44, -54, $-61,-70,-72,-81$ ) and 18 HR-HPV or probably HR-HPV (HPV-16, -18, -26, $-31,-33,-35,-39,-45,-51,-52,-53,-56,-58,-59,-66,-68,-73,-82)$.

Samples that were positive by gel electrophoresis but negative in reverse line blot for any of the identifiable types were considered as HPV undetermined.

\section{Results}

HPV DNA was detected in two hundred and twenty-two patients (70.5\%) distributed differently in the cytological categories: the HPV prevalence estimates were 50\% (48/96) in women with ASCUS, $80.8 \%$ $(126 / 156)$ in women with L-SIL, and 76.2\% (48/63) in women with H-SIL.

Table 1 shows the distribution of the results of the HPV detection related to cytological abnormalities.

Multiple HPV infection was found in $35.1 \%$ of the HPV DNA positive subjects; in particular, $31.2 \%$ of ASCUS, $40.5 \%$ of L-SIL, and $25 \%$ of the H-SIL groups patients were infected by two or more HPV genotypes.

Among the 222 HPV DNA positive patients, the prevalence of HRHPV genotypes increased with the severity of cytology: 36 (75.0\%) ASCUS and 104 (82.6\%) L-SIL women were positive for HR-HPV, with multiple infections in 15 and 51 cases, respectively. In all H-SIL women were detected HR-HPV, with multiple infections in 12 cases: 9 in combination with at least another HR-HPV and 3 with LR-HPV.

HPV 16 was the most prevalent genotype in all the categories considered: $31.3 \%(15 / 48)$ of ASCUS, $52.4 \%(66 / 126)$ of L-SIL, and $93.5 \%$ (43/48) of H-SIL.

Table 1. Distribution of human papillomavirus DNA detection related to cytological abnormalities.

\begin{tabular}{lccc} 
& ASCUS, n. (\%) & L-SII, n. (\%) & HIL, n. (\%) \\
Negative & $48(50.0)$ & $30(19.2)$ & $15(23.8)$ \\
HR S.I. & $21(43.8)$ & $53(42.1)$ & $36(75.0)$ \\
\hline LR S.I. & $7(14.6)$ & $13(10.3)$ & 0 \\
UD & $5(10.4)$ & $9(7.1)$ & 0 \\
\hline M.I. & $15(31.2)$ & $51(40.5)$ & $12(25.0)$ \\
Total & $96(30.5)$ & $156(49.5)$ & $63(20.0)$ \\
\hline
\end{tabular}

ASCUS, atypical squamous cells of undetermined significance; L-SIL, low grade squamous intraepithelial lesion; H-SIL, high grade squamous intraepithelial lesion; SI, single infection; UD, undetermined; MI, multiple infections. 
HPV 18 was detected in 27 patients (12.2\%), in association with other genotypes in 21 cases.

After HPV 16 and 18, the more prevalent genotypes were HPV 31 (9.5\%) and HPV 6 (9.5\%) in all the categories considered (Table 2).

\section{Discussion and Conclusions}

In this study, we observed the distribution of different HPV genotypes in a cohort of 315 women from Eastern Sicily.

HPV was detected with high frequency not only in women with HSIL, but also in women with low-grade lesions (ASCUS and L-SIL).

HPV 16 was the genotype most frequently detected in all studies reported in literature in either single or multiple infections with other genotypes.

Owing to its association with more than $50.0 \%$ of all cervical cancers (22), the prevalence of HPV 16 in the female populations gains particular interest. Compared to other HPV types, the frequency of detection of HPV 16 can differ among countries (26). In the present study HPV 16 was the more common HR-HPV (56.7\%) revealed with a prevalence increasing with the severity of abnormal cervical cytology (31.3\% in ASCUS, $52.4 \%$ in L-SIL, and 93.5\% in H-SIL).

HPV 16 is a persistent genotype, it is highly carcinogenic, and it can be integrated into the host genome, becoming one of the major contributing factors to genital malignant transformation $(14,18)$.

From our results it appears that HPV 16 (56.7\%), 18 (12.2\%), 6 $(9.5 \%)$, and $31(9.5 \%)$ were the most prevalent genotypes followed by HPV 52 (6.8\%), and 59 (6.8\%).

HPV 45, considered as the third most common genotype in invasive cervical cancer, was never found in the examined positive women.

HR-HPV positivity rates of $75.0 \%, 82.6 \%$ and $100 \%$, found in women with ASCUS, L-SIL and H-SIL respectively, in the present study are largely comparable with those reported in the literature $(8,25,1)$.

Moreover, the high frequency of detection of HR-HPV in ASCUS group, suggests to screen also the ASCUS cases for HPV DNA. Finally, the finding of HPV 16 positivity in $93.7 \%$ of the H-SIL cases, in either single or multiple infection, suggests that the probability of progression from ASCUS to H-SIL or invasive cancer in women infected with HPV 16 may be quite high and so far underestimated. As reported by Anderson (2), the sensitivity of the HPV DNA test is higher than a repeated Pap smear; thus HPV testing had a very high negative predictive value (99.5\%) in women with an ASCUS Pap smear. This indicates that if an HPV test is negative, cervical disease due to HPV is highly unlikely. These observations support HPV DNA testing as viable option in the management of women with ASCUS cytology.

The role and the impact of multiple HPV infections on the risk of cervical dysplasia is controversial $(9,13,20)$. Becker et al. (4) suggested that multiple HPV infections carry an increased risk of dysplasia only in patients with HPV 16. We observed that multiple infections are common and the proportion of multiple infections decreases in H-SIL (25.0\%) if compared with ASCUS (31.2\%) and L-SIL (40.5\%), as reported by Agarossi et al. (1).

This observation reinforces the theory of the multi-stage cancer model, by which one HPV type tends to predominate with the rise of cervical lesion severity (23).

Further studies are on-going to define the role of HPV DNA testing in the management of cervical disease due to HPV infection.

Although the Pap test is an important diagnostic tool in cervical-vaginal screening for its high sensibility, it has the disadvantage of not being able to reveal the infection with HR-HPV in the absence of cytological abnormalities.

Current molecular technologies, including HPV DNA detection and the E6/E7 mRNA test, which provides important information about the potential progression of HPV infection, represent a more accurate, sensitive and specific, diagnostic approach as well as a more valid method for posttreatment follow-up of women with significant cytological abnormalities.

In this way the pre-cancerous lesions can be identified and treated precociously in order to lessen the physical and psychological impact on women.

Table 2. Prevalences of the different genotypes in the cytological categories.

\begin{tabular}{|c|c|c|c|c|}
\hline Genotypes & ASCUS $(50.0 \%)$ & L-SIL (80.8\%) & H-SIL (76.2\%) & TOT. (70.5\%) \\
\hline 6 & 11.1 & 14.3 & - & 9.5 \\
\hline 40-42-69 & 11.1 & 7.1 & - & 5.4 \\
\hline $43-61-70$ & 11.1 & 9.5 & - & 6.8 \\
\hline 16 & 31.3 & 52.4 & 93.5 & 56.7 \\
\hline 18 & 18.7 & 9.5 & 12.5 & 12.2 \\
\hline 31 & 18.7 & 9.5 & - & 9.5 \\
\hline 33 & - & 4.8 & $6.2 \%$ & 4 \\
\hline 35 & - & - & $6.3 \%$ & 1.3 \\
\hline 39 & 11.1 & 2.9 & $6.2 \%$ & 4 \\
\hline 51 & - & 2.9 & - & 1.3 \\
\hline 52 & 11.1 & 9.5 & - & 6.8 \\
\hline 53 & 11.1 & 7.1 & - & 5.4 \\
\hline 56 & - & 4.8 & - & 5.4 \\
\hline 58 & 11.1 & 7.1 & - & 5.4 \\
\hline 59 & 11.2 & 9.5 & - & 6.8 \\
\hline 66 & 11.1 & 4.8 & - & 4 \\
\hline 73 & - & 7.1 & - & 4 \\
\hline U.D. & 12.5 & 7.1 & - & 6.8 \\
\hline
\end{tabular}

ASCUS, atypical squamous cells of undetermined significance; L-SIL, low grade squamous intraepithelial lesion; H-SIL, high grade squamous intraepithelial lesion; UD, undetermined. 


\section{References}

1. Agarossi A, Ferrazzi E, Parazzini F, et al. Prevalence and type distribution of high-risk human papillomavirus infection in women undergoing voluntary cervical cancer screening in Italy. J Med Virol 2009;81:529-35.

2. Anderson SM. Human papillomavirus and cervical cancer. Clin Microbiol News 2002;24:113-8.

3. Baseman JG, Koutsky LA. The epidemiology of human papillomavirus infections. J Clin Virol 2005;32:16-24.

4. Becker TM, Wheeler CM, McGrough NS, et al. Sexually transmitted diseases and other risk factors for cervical dysplasia among Suothwestern Hispanic and non-Hispanic white women. JAMA 1994;271:1181-8.

5. Bosch FX, Muñoz N. The viral etiology of cervical cancer. Virus Res 2002;89:183-90.

6. Centurioni MG, Puppo A, Merlo DF, et al. Prevalence of human papillomavirus cervical infection in an Italian asymptomatic population. BMC Infect Dis 2005;5:77.

7. Clifford G, Smith J, Aguado T, Franceschi S. Comparison of HPV type distribution in high-risk cervical lesions and cervical cancer: a meta-analysis. Br J Cancer 2003;89:101-5.

8. Clifford GM, Gallus S, Herrero R, et al. IARK HPV prevalence surveys study group. Wordlwide distribution of human papillomavirus types in cytologically normal women in the International Agency for Research on Cancer HPV prevalence surveys: a pooled analysis. Lancet 2005;366:991-8.

9. Cuschieri KS, Cubie HA, Whitley MW, et al. Multiple high risk HPV infections are common in cervical neoplasia and young women in a cervical screening population. J Clin Pathol 2004;57:68-72.

10. Cuschieri KS, Whitley MJ, Cubie HA. Human papillomavirus type specific DNA and RNA persistence--implications for cervical disease progression and monitoring. J Med Virol 2004; 73:65-70.

11. De Francesco MA, Gargiulo F, Schreiber C, et al. Detection and genotyping of human papillomavirus in cervical samples from Italian patients. J Med Virol 2005;4:588-92.

12. de Sanjosè $S$, Diaz $M$, Castellsagué $X$, et al. Worldwide prevalence and genotype distribution off cervical human papillomavirus DNA in women with normal cytology: a meta-analysis. Lancet Infect Dis 2007;7:453-9.

13. Fife KH, Cramer HM, Schroeder JM, Brown DR. Detection of multiple human papillomavirus types in the lower genital tract correlates with cervical dysplasia. J Med Virol. 2001;64:550-9.

14. Gallo G, Bibbo M, Bagella L, et al. A study of viral integration of
HPV-16 in young patients with LSIL. J Clin Pathol 2003;56:532-36.

15. Gargiulo F, De Francesco MA, Schreiber C, et al. Prevalence and distribution of single and multiple HPV infection in cytological abnormal cervical samples from Italian women. Virus Res 2007;125:176-82.

16. Ho L, Terry G, Londesborough P, et al. Human papillomavirus DNA detection in the management of women with twice mildly abnormal cytological smears. J Med Virol 2003;69:118-21.

17. Kulasingam SL, Hughes JP, Kiviat NB, et al. Evaluation of human HPV testing in primary screening for cervical abnormalities: comparison of sensitivity, specificity of frequency of referral. JAMA 2002;288:1749-57.

18. Liu SS, Tsang PC, Chan KY, et al. Distribution of six oncogenic types of human papillomavirus and type 16 integration analysis in Chinese women with cervical precancerous lesions and carcinomas. Tumor Biol 2008;29:105-13.

19. Rassu M, Bertoloni G, Mengoli C, et al. HPV genotype prevalence in cervical specimens with abnormal cytology: a report from northeast Italy. Scand J Infect Dis 2005;37:476-81.

20. Rolòn PA, Smith JS, Muñoz N, et al. Human papillomavirus infection and invasive cervical cancer in Paraguay. Int $\mathrm{J}$ Cancer 2000;85:486-91.

21. Ronco G, Ghisetti V, Segnan N, et al. Prevalence of human papillomavirus infection in women in Turin, Italy. Eur J Cancer 2005; 41:297-305.

22. Schiffman M, Castle PE, Jeronimo J, et al. Human papillomavirus and cervical cancer. Lancet 2007;370:890-907.

23. Schiffman M, Herrero R, Desalle R, et al. The carcinogenicity of human papillomavirus types reflects viral evolution. Virology 2005;337:76-84.

24. Selva L, Gonzalez-Bosquet E, Rodriguez-Plata MT, et al. Detection of human papillomavirus infection in women attending a colposcopy clinic. Diagn Microbiol Infect Dis 2009;64:416-21.

25. Smith JS, Lindsay L, Hoots B, et al. Human papillomavirus type distribution in invasive cervical cancer and high-grade cervical lesions: a meta-analysis update. Int J Cancer 2007;121:621-32.

26. Smith JS, Melendy A, Rana RK, Pimenta JM. Age-specific prevalence of infection with human papillomavirus in females: a global review. J Adolesc Health 2008;43:S5-25.

27. Tornesello ML, Duraturo ML, Botti G, et al. Italian HPV Working Group: prevalence of alpha-papillomavirus genotypes in cervical squamous intraepithelial lesions and invasive cervical carcinoma in the Italian population. J Med Virol 2006;78:1663-72.

28. zur Hausen H. Papillomavirus and cancer: from basis studies to clinical application. Nat Rev Cancer 2002;2:342-50. 